\title{
Contexto, justicia y universalidad en la filosofía política actual. Algunas críticas y aportes a la teoría de la justicia de Habermas*
}

\author{
Ricardo Florentino Salas Astrain (Chile)**
}

\section{Resumen}

A partir del giro pragmático de la filosofía contemporánea, las teorías de la justicia han avanzado en una serie de temas relativos a la justificación de los principios de justicia y que presuponen el nexo entre universalidad y particularidad histórico-cultural. Se critica la apelación únicamente a los principios racionales y abstractos, ya que se dejan de lado las virtualidades de los contextos que aglutinan las experiencias morales de pueblos, comunidades de vida y movimientos sociales en una sociedad globalizada. Si las teorías discursivas de la justicia requieren articular tal nexo la cuestión teórica es encontrar la veta que permita vincular procedimentalismo y substantividad histórica. Por lo tanto, ¿en qué sentido la teoría habermasiana de la justicia exigiría un replanteamiento teórico explícito acerca de la capacidad normativa del contexto? La hipótesis propuesta aquí revela la respuesta de Rainer Forst y Mark Hunyadi, ya que estos replantean una teoría contextual de la justicia y reelaboran una noción filosófica de contexto que demuestra nuevas posibilidades y limitaciones de la teoría discursiva de la justicia.

\section{Palabras clave}

Filosofía Política; Teoría Política; Teoría de la Justicia; Teoría Crítica; Escuela de Frankfurt.

Fecha de recepción: mayo de 2018 • Fecha de aprobación: noviembre de 2018

\section{Cómo citar este artículo}

Salas Astrain, Ricardo Florentino. (2019). Contexto, justicia y universalidad en la filosofía política actual. Algunas críticas y aportes a la teoría de la justicia de Habermas. Estudios Políticos (Universidad de Antioquia), 55, pp. 163-181. http://doi.org/10.17533/udea.espo.n55a08

\footnotetext{
* Artículo elaborado en el marco del proyecto de investigación del Fondo Nacional de Desarrollo Científico y Tecnológico (Fondecyt), Chile, N. ${ }^{\circ} 1170383$, Problemas y perspectivas de una teoría contextual de la justicia, 2017-2021.

** Profesor, magíster y doctor en Filosofía. Docente de la Facultad de Ciencias Sociales y Humanidades, Universidad Católica de Temuco, Chile. Correo electrónico: rsalas@uct.cl - Orcid: https://orcid. org/0000-0003-4765-1567
} 


\title{
Context, Justice and Universality in Current Political Philosophy. Some Critiques and Contributions to the Habermas's Theory of Justice
}

\begin{abstract}
From the pragmatic turn of contemporary philosophy, the theories of justice have advanced in a series of topics related to justifying the principles of justice which presuppose the connection between universality, and historical and cultural particularity. The exclusive appeal to rational and abstract principles is criticized, since it sets aside the implicit influence of contexts, which unite the moral experiences of peoples, communities of life, and social movements in a globalized society. If discursive theories of justice require to articulate this connection, the theoretical challenge is to find the joint that would allow to link historical proceduralism and substantivity. In which sense the Habermasian theory of justice would demand an explicit theoretical reconsideration about the normative capacity of context? The article proposes that an answer to this problem can be found in the works of R. Forst and M. Hunyadi, since they rethink a contextual theory of justice and restore a philosophical notion of context which demonstrate new possibilities and limitations of the discursive theory of justice.
\end{abstract}

\section{[164] Keywords}

Political Philosophy; Political Theory; Theory of Justice; Critical Theory; Frankfurt School. 
Contexto, justicia y universalidad en la filosofía política actual. Algunas críticas...

\section{Introducción}

La idea y la cuestión de la justicia refieren en la filosofía política actual a una pluralidad de teorías y culturas filosóficas expresadas en proyectos disímiles de gran densidad y precisión conceptual, lo que se puede ilustrar con importantes propuestas de justificación de los principios de justicia. Las obras elaboradas por John Rawls (1971) y Jürgen Habermas (1992) renovaron singularmente el campo filosófico contemporáneo, lo que se demuestra en la enorme cantidad de estudios al respecto y de las sucesivas publicaciones de discípulos y críticos que prosiguen estas ideas a los dos lados del Atlántico, tales como Axel Honneth, Amartya Sen, León Olivé Morett, Nancy Fraser, Gustavo Pereira, Rainer Forst y Mark Hunyadi (Pereira, 2013a). Estos dos últimos filósofos guiarán este artículo para cuestionar la fuerza del contexto en la teoría política actual.

Es de algún modo obvio que no existe una sola teoría normativa de la justicia, lo que está ligado en buena medida a los disímiles diagnósticos, puntos de partida, principios normativos realzados y al modo de justificación que es posible encontrar en cada una de ellas. En suma, este artículo sigue la perspectiva de que hay que distinguir entre una teoría empírica o general de la justicia, con sus variantes, y un enfoque normativo sobre ella. Por «normativo»se entiende aquí la dimensión ético-social/ético-política de la justicia, o también el rasgo filosófico-normativo de ella. Se diferencia tanto de una teoría instrumentalista —ocupada como medio, en función de acortar distancias sociales o disminuir brechas en el acceso a bienes sociales fundamentales-, como de una postura neoliberal, para la cual no es factible hablar de justicia en sentido social, sino solo de lo justo en el orden legal de las cosas dadas (Salas, 2015).

Para esclarecer lo que está en el centro de este trabajo, se señala lo que se entiende por «contexto» en una primera aproximación. Habermas (2000) plantea en un corto pasaje si los principios de justicia fundamentados reconstructivamente valen para las sociedades por sus tradiciones políticoculturales o bien para situaciones y condiciones de la vida moderna en general y, en consecuencia, discute si se puede defender la tesis de la primacía de lo justo sobre lo bueno a partir de las distinciones entre las interpretaciones del mundo moderno o las imágenes del mundo fundamentalista. 
En otra parte, Habermas (1992) reprocha las limitaciones teóricas que supone partir de una tradición política específica: «Rawls tendría que admitir entonces que ambos principios de justicia no pueden pretender validez alguna para los alemanes porque en la cultura e historia alemanas no pueden encontrarse equivalentes de la tradición constitucional americana que deban considerarse efectivos en lo que a formación y a educación políticas se refiere» (Habermas, 1996a, p. 127). En esta controversia se alude a que una teoría de la justicia tendría que fundarse sobre principios que no se definen por situaciones histórico-culturales, y que éstas, al parecer, no pueden nunca proporcionar criterios para la validez de los principios en que reposa tal teoría por ser plural e histórica. Un contexto refiere entonces a tales experiencias morales de sujetos y colectividades.

Forst (1994) plantea una tesis general donde pretende religar la cuestión del universalismo propio de la justicia con los contextos de vida a partir del debate teórico entre lo contextual y lo procedimental, tal como aparece en el debate de los autores norteamericanos denominados «comunitarios» y «liberales». Trabajando los respectivos conceptos de justicia — Ronald Dworkin, John Rawls, Charles Taylor, Michael Sandel, Michael Walzer-y asumiendo la propuesta discursiva de Habermas concluye que en dicha controversia se destacan cuatro cuestiones centrales: la constitución del yo, la neutralidad ética de la ley, el ethos de la democracia y el que lleva a la cuestión más relevante, el vínculo entre los principios universales y las dinámicas históricas — contextualismo—. Forst termina afirmando que una teoría de la justicia que no sea unilateral debe tener en cuenta estos cuatro contextos de justicia. Sobre esta base, los argumentos liberales y comunitarios pueden compartirse con otros, como los enfoques teóricos feministas o bien discursivos, ya que en todos ellos hay acepciones diferenciadas respecto de lo que se entiende por persona y comunidad.

El filósofo suizo Mark Hunyadi, traductor de parte de la obra moral de Habermas al francés, considera también que la cuestión del contexto aparece siendo la noción central desde la perspectiva de una teoría moral y de una filosofía social y política. Indica que la noción de contexto ha sido olvidada como fuente (source) y recurso (ressource) en las teorías morales y políticas contemporáneas. Arguye que los contextualistas como Richard Rorty o los anticontextualistas como Habermas «comparten un concepto esencialmente pobre de contexto asimilado en grueso a las formas wittgenstenianas de vida, lo que los condena idénticamente a hacer un uso acrítico de ella» (Hunyadi, 
2009, p. 319). ${ }^{1}$ Para él, es preciso hacer frente a la teoría moral habermasiana que pretende expurgar las referencias a lo contextual y a lo empírico.

En síntesis, con estas referencias a Habermas, Forst y Hunyadi dejan en evidencia que un contexto cabe entenderlo como la posición que asumen los actores en sus respectivos locus y que, por lo tanto, la experiencia que ellos tienen de sus vicisitudes históricas, de sus mundos de vida, y de sus memorias de injusticia constituyen el carácter histórico cultural mismo de las experiencias morales. La dificultad radica en que una concepción de las experiencias de los sujetos, en especial de las experiencias de injusticia (Renault, 2004), pueden tener cabida al interior de cualquier teoría de la justicia, y dejan instalada, al menos eventualmente, la interrogante de si una teoría contextual de la justicia únicamente puede justificar en un sentido fuerte solo apelando a principios racionales o requiere necesariamente establecer formas de mediación a la experiencia moral.

A partir de esta noción, las ideas desplegadas por Forst y Hunyadi en sus diferentes trabajos filosóficos de los últimos años refieren a un particular modo de articular una teoría universal de la justicia con los contextos históricoculturales. En especial las ideas de Hunyadi, menos conocidas en castellano, son relevantes ya que propone un concepto de contextualismo fuerte. En L'homme en contexte (Hunyadi, 2012) expone que la tarea filosófica principal es demostrar que los seres humanos están en contexto durante toda su vida, y por lo mismo es preciso cuestionar las teorías de la justicia que no asumen este carácter porque en principio se sigue suponiendo que el orden experiencial e histórico tiene que ser pensado a partir de una racionalidad que solamente puede establecer la validez.

Este trabajo parte del diferendo acerca de lo que significa pensar los contextos en el marco de una teoría de la justicia que prosigue la senda habermasiana. Con y contra Habermas, Hunyadi piensa que la contextualidad es la primera condición del ser humano. Este olvido teórico de la «experiencia moral», tal como la viven los propios actores, ha conducido a olvidar la fuerza contrafáctica de los contextos. La controversia entre el modo de sostener un contextualismo fuerte por Hunyadi conduce a pensar en sus diferencias con la obra de Forst. Se cuestiona así los criterios críticos, si ya están implícitos en el contexto, en su totalidad o en parte. En suma, la interrogante que se

${ }^{1}$ Todas las citaciones en castellano de los textos de Mark Hunyadi son de responsabilidad del autor de este artículo. 
pretende responder es si el contexto es algo que debería ser trascendido para poder alcanzar la crítica o la reflexión — como nos enseña una cierta versión del universalismo- o si la situación cultural de la época debe ser considerada como una dimensión constitutiva de la moral y de la política.

\section{El telón de fondo del debate acerca de la teoría habermasiana de la justicia}

En el centro de la cuestión contextual aludida, de un modo u otro se plantea la cuestión entre racionalidad, normatividad y contingencia. Este debate confronta un estilo de pensar la justicia que proviene del kantismo y del modo en que este ha sido reasumido en el planteo de los filósofos frankfurtianos y en el proyecto rawlsiano.

Por una parte, se puede indicar que el legado kantiano en la ética discursiva tiene que ver —en primer lugar- con el quiebre que se establece al interior de una philosophia prima, por la cual el modelo kantiano se ubica aun en el terreno de una crítica de la razón entendida como filosofía de la conciencia, que incluso alcanzaría hasta Edmund Husserl (Hunyadi, 2009). En esta forma de filosofar no se asume todavía el desafío de una filosofía

[168] del lenguaje, la cual permita una conceptualización que justifique lo que Habermas ha hecho conocido como como «acción comunicativa».

Por otra parte, dichas controversias refieren a un cuestionamiento del modo en que la razón pura práctica se fue ensamblando con la razón pura teórica, donde la simetría que se propone entre una y otra es discutible. Por último, también refiere a una concepción cada vez más diferenciada del ámbito de lo político, ético y jurídico que ha sido medular en las sociedades modernas. La crítica actual a Kant se hace a partir de una reactualización de algunos de sus nudos teóricos. En este sentido, el neokantismo habermasiano permite proponer una noción de racionalidad comunicativa en el marco de una filosofía del lenguaje, en el que la razón práctica refiere a las articulaciones institucionales propias de la sociedad moderna.

Tal herencia kantiana no es solo confrontada por Habermas sino también por Rawls y es madurada también en los trabajos de Honneth, Forst y Hunyadi. A pesar de las grandes diferencias, los dos últimos, empero, poseen varios aspectos comunes — como lo afirma Habermas-, como los que se dan entre miembros que pudiendo diferir en una serie de aspectos y que pertenecen a una misma familia teorética (Forst, 2014). 
En síntesis, los planteamientos de Habermas y Rawls son poskantianos y posmetafísicos, ambos son críticos del utilitarismo, uno y otro rechazan el irracionalismo ético, sea que provenga del decisionismo o del emotivismo; por lo mismo, ciertamente hay entre ellos puntos de convergencia teórica que no se pueden ignorar.

Sin embargo, en tres puntos divergen: el primero concierne al método para obtener acuerdos normativos inclusivos y participados. Para Habermas, Rawls permanece anclado en el paradigma de la conciencia, ligado a un sujeto solitario y solipsista — velo de ignorancia y posición original—, es decir, que no hace el tránsito a un paradigma de la intersubjetividad en la fundamentación de principios o normas. El segundo punto es la crítica que dirige Habermas al modo rawlsiano de plantear las conexiones entre autonomía privada y autonomía pública en el campo del ejercicio de la ciudadanía. Una forma demasiado esquemática y poco dinámica en cuanto al lugar que tiene el diálogo entre participantes y la mediación del derecho en el logro de instituciones justas. El tercer punto se refiere a que Habermas trata más bien de dibujar el ideario de una democracia radical en el cual desempeña un rol central una idea de justicia democrática y no la precisión de principios que se limiten a regular el terreno de la igualdad-desigualdad.

Al focalizar los entretelones de una teoría habermasiana de la justicia se vislumbra, desde el primer momento, que en el ejercicio de la ciudadanía y el espacio privado se da una dialéctica, la cual a través del uso público de la razón y la praxis argumentativa pueden reconstruir los consensos de manera pública y colectiva por parte de todos los participantes. En orden a establecer una sociedad justa, él piensa que es el consenso argumentativo, abierto y participativo por los cuales no solo hay principios en juego sino también una co-participación en la génesis de una juridicidad de la cual los ciudadanos pueden entenderse como autores (Rawls y Habermas, 1998, pp. 66 y ss.). Los principios de justicia se logran entonces a través de una racionalidad deliberativa y mediante ella la «fuerza del mejor argumento».

Hunyadi (2015a) ha resumido bien esta diferencia desde el punto de vista de los actores, la cual se refiere a la relevancia del contexto de acción de una comunicación con sentido:

Pero la diferencia fundamental con el modelo de Habermas es que los actores rawlsianos solo razonan de una manera descontextualizada sobre los principios de justicia que se definen en otra parte, ahí donde 
los actores habermasianos parten de su experiencia comunicacional y descubren, de forma inmanente a esta experiencia, los criterios de legitimidad de los estándares sobre los que podrían estar de acuerdo (p. 81).

Para Habermas los principios determinados de justicia suponen siempre una noción implícita de justicia. Tal óptica presupone el logro de ciertas condiciones de justicia, entendida como libertad activa y como capacidad de ejercicio de derechos en igualdad de condiciones, y con respeto a la condición humana. Por lo tanto, el mayor peso de su concepción está puesto en el ejercicio de una racionalidad comunicativa y en los procedimientos a través de los cuales se legitiman normas, leyes e instituciones, por medio de la opinión y voluntad política de los ciudadanos.

Tales ideas, empero, no van de suyo, Pereira (2013b) propone elementos clave para la elaboración de una crítica de la ética del discurso que permita comprender cómo desde Teoría de la acción comunicativa Habermas ha logrado avanzar hasta Facticidad y validez para articular el concepto de justicia, democracia y derecho en el Estado moderno. ${ }^{2}$

[La teoría democrática de Habermas] pretende garantizar la necesaria cohesión social la que debe presentarse de tal modo que pueda ser compartida por todos los ciudadanos, cualesquiera que sean sus creencias que profesen y los modos de vida que sigan. Eso no significa, empero, que en los asuntos éticos - las cuestiones referentes a la identidad personal y las concepciones del bien $-y$, sobre todo, las morales — relativas a la cuestión de la justicia social— no deban ser objeto de discusión pública, sino tan solo que las condiciones y presupuestos de los procedimientos de deliberación y toma de decisión sobre tales cuestiones deben ser estrictamente neutrales con respecto a las visiones particulares del mundo (Velasco, 1999, p. 21).

Esta indicación es central porque para Habermas la opinión pública democrática requiere la discusión de las visiones particulares, pero esto no puede suplantar procedimientos que deben ser plenamente racionales y, por lo tanto, neutrales. Entonces, el esfuerzo habermasiano se puede justipreciar en cuanto contribuye a esclarecer los sentidos de la justicia y la democracia en

\footnotetext{
${ }^{2}$ Esta discusión refiere específicamente al capítulo 2 (Habermas, 1996b). También en Die Einbeziehung des Anderen (1996a), texto en el que Habermas retoma y aclara las críticas suscitadas por Faktizität und Geltung (1992).
} 
el Estado democrático de derecho en las sociedades modernas. Una teoría de la democracia basada en una concepción de la «acción comunicativa» logra avanzar en la elaboración de elementos que permitan profundizar el ejercicio liberal democrático actual y no en vistas de una «democracia radical».

Cabe concluir que la «teoría de la justicia» de Habermas hace referencias específicas acerca del sentido de la reconstrucción crítica del modelo del contrato social y desarrolla un constructivismo en la generación de los principios de justicia. Pero en Habermas tales principios siempre se construyen: «En suma: el problema teórico de la fundamentación se desplaza, pasando de las propiedades del procedimiento a las características de las personas» (Habermas, 2000, p. 137).

Por todo lo ya indicado, queda en evidencia que la cuestión misma del contexto es ya parte de una de las cuestiones disputadas de una teoría discursiva de la justicia, y los aportes críticos que se verán a continuación intentan delinear solo algunas de las principales dificultades, la modalidad como son enfrentadas o contorneadas por Forst y Hunyadi, cuando no de intentar superarlas apelando a otros recursos teóricos provenientes de fuera del neokantismo, y que se proyectan renovando el estatus de la filosofía social y de la filosofía política.

\section{Forst y Hunyadi. Lecturas entrecruzadas sobre la cuestión del contexto en la teoría discursiva de la justicia}

Se asumirán ahora algunos aspectos problemáticos de la teoría habermasiana de la justicia y, en particular, su profundización de la noción de contexto que se encuentran explícitamente referidos a los planteos de Forst y de Hunyadi.

Forst (1994) plantea una tesis en la que, asumiendo la propuesta discursiva de Habermas, religa la cuestión de la justicia con el debate sobre universalismo y contextualismo en el mundo anglosajón. En dicho estudio, Forst analiza los debates las teorías de justicia de liberales y comunitarios, y los resume en cuatro problemas centrales de la filosofía política: a) la cuestión de la constitución del «yo»; b) la neutralidad de los principios generales de la ley y la justicia sobre las concepciones del bien; c) el poder integrador de las visiones postradicionales y democráticas de la comunidad política; y d) la crítica contextualista de las teorías morales universalistas. 
Como ya se indicó, su tesis determina que una correcta teoría de la justicia, no unilateral, requiere comprender cuatro contextos de justicia en relación con las nociones de «persona» y «comunidad». Sobre esta base, no es preciso oponer los argumentos liberales contra los de comunitarios, ya que aquellos pueden compartirse con enfoques teóricos de variado tipo. Tal reconstrucción relativa al sujeto o a la persona obliga a pensar de otra manera el debate teórico entre procedimentalismos y contextualismos, así como ya aparece en los autores angloamericanos - trabajados en su tesis doctoral-, y le hace avanzar hacia nuevas consideraciones sobre los conceptos de justicia en uso.

En Kritik der Rechtfertigungverhaltnisse —-dedicado a HabermasForst (2011) postula un ideal discursivo de la justicia que él vincula con la cuestión central de la filosofía política, que no puede ser otro que el de la justificación: «La primera pregunta de la justicia es la que inquiere acerca de la distribución del "poder de justificación" en una sociedad, sabiendo que éste no es un "bien" de fácil distribución» (2014, p. 26). La hipótesis elaborada por Forst propone conectar el principio de la justificación con un marco de un pluralismo diagnóstico y evaluativo (pp. 26-27).

[172 ] Forst (2014, pp. 129-147) explícita su posición en divergencia con otros colegas germanos y norteamericanos. Comentando lo que denomina «las dos figuras de justicia» en la tradición filosófica actual, considera que Fraser y Honneth representan dos intentos de construir una amplia teoría crítica de la justicia, y que en dicho debate evocan un clásico «cisma de la crítica» que refiere al discurso de la ilustración y a la tradición marxista (p. 129). Sobre este punto álgido de litigio afirma que: «El lenguaje primordial de la crítica era y es el lenguaje del poder y del pedido de razones de justificación para las normas y las instituciones a las que están sometidos todos, no en primer lugar el lenguaje del reconocimiento en un sentido más sustantivo» (p. 170). Si el lenguaje del poder es lo que está en juego, la cuestión relevante es indagar qué acontece con la justicia en contextos de asimetría y con los ideales de una vida autorrealizada:

La primera crítica se basa en una idea de justicia y tiene como meta el establecimiento de las relaciones sociales y políticas sin asimetrías de poder profundas y formas de dominio injustificadas; la segunda línea, en cambio, utiliza como herramientas de la crítica conceptos cualitativos, éticamente sustantivos, autorrealización, «verdadera», una 
Contexto, justicia y universalidad en la filosofía política actual. Algunas críticas...

forma de vida «con sentido» o «estar a buen recaudo» en las distintas formas del reconocimiento mutuo y de la vida social (p. 130).

Forst (2014) considera así que ambas concepciones derivan de dos tradiciones diferentes de la crítica: una de índole kantiana y la otra hegeliana. Su conclusión acerca de la definición correcta de una teoría crítica de la justicia es que ella tiene que evitar determinar las dificultades de la fundamentación normativa y de la perspectiva que tiene la teoría crítica sobre las sociedades de nuestro tipo —y más allá de ellas_- Esto le lleva a postular una tercera propuesta que, en sus palabras, «Lo he llamado enfoque de lo más importante, primero o, expresado técnicamente, monismo de la justificación y pluralismo diagnóstico-evaluativo» (p. 134), lo que se basa en el principio de la justificación, pero en el marco de un pluralismo diagnóstico y evaluativo (pp. 26-27).

Este tercer enfoque, alternativo a la teoría crítica, contiene una idea clave para elaborar un proyecto renovado de la teoría crítica que, sin desprenderse de su arraigo habermasiano, puede dialogar con la teoría del reconocimiento de Honneth, reconociendo la capacidad de ésta para dar cuenta de las experiencias de sufrimiento social, en general, y para la injusticia, en sentido estricto. Sin embargo, si se requiere justificar las pretensiones de justicia es indispensable «un enfoque procedimental-deontológico de una teoría del discurso» (Forst, 2014, p. 134).

En términos de la actual discusión, este asunto es relevante ya que Forst busca consolidar una vía que, aceptando el basamento habermasiano, no le lleve a desconocer lo que Honneth ha intentado probar, esto quiere decir que debería circunscribirse a un enfoque procedimental de la justicia con componentes sustantivos.

La justicia no se refiere primordialmente a lo que alguien tiene (o no tiene) sino, en primer lugar, a cómo es tratado. La justicia no es un concepto teleológico, dado que, en primer lugar, se sustenta en deberes deontológicos respecto de aquello que las personas se deben unas a otras dentro de un contexto de justicia; y en segundo lugar, la mirada no se dirige a lo que les falta a las personas y sería bueno para ellas, sino más bien que a las personas se las priva de algo a lo que pueden aspirar como razones recíprocas y generales no refutables. La justicia tiene como meta terminar la dominación y el ejercicio del poder no justificado y arbitrario (Forst, 2014, p. 135). 
A partir de estas ideas sobre la justicia, Forst propone una afirmación central para la filosofía política que se resume en lo siguiente:

La filosofía política comienza con la pregunta misma de la justificación, pero una filosofía política reflexiva y crítica la dirige hacia sí misma: ¿qué quiere decir plantear y contestar la pregunta acerca de la justificación, qué es lo que se requiere para tal fin y qué condiciones hay implícitas en las tesis de que una justificación tal tiene que ser «razonable», «aceptable», «justa» (Forst, 2014, p. 16).

En otras palabras, aunque el problema filosófico radicaría en determinar adecuadamente la práctica de la justificación, su enfoque de la política intenta superar la dicotomía instalada entre el procedimentalismo y el sustancialismo; se trata, como lo dice él mismo, de «reconstruir recursivamente las normas, algunas de naturaleza procedimental, otras de naturaleza sustantiva, que posibilitaron tal justificación» (Forst, 2014, p. 19).

Forst piensa que una concepción crítica de la justificación tiene al menos cinco tareas fundamentales como teoría política: a) proponer un análisis crítico de la sociedad que indique las relaciones sociales y políticas que no son justificables; b) hacer una crítica genealógica de las justificaciones falsas de las relaciones asimétricas; c) exigir como primer imperativo de la justicia relaciones sociales y políticas justificadas; d) plantear una explicación del fracaso de las estructuras sociales y políticas para desenmascarar relaciones injustificadas; e) identificar patrones de su actividad crítica por la que se vincula toda pretensión de validez con la posibilidad de que los sometidos a las normas las aprueben.

Las derivaciones teóricas para una teoría de la justicia son claras: «la justicia no es solo lo que vale como justo en una sociedad sino lo que debería valer como recíproco y general si los sometidos a las normas fueran autores de las mismas, libres e iguales. Que se conviertan en esto es la primera exigencia de la justicia» (Forst, 2014, p. 19). En este sentido, el reconocimiento es el verdadero punto álgido de este difícil diálogo para hacer frente a la construcción de una teoría normativa de la justicia, en diálogo y disputa con Habermas y con Honneth (Sauerwald y Salas, 2017).

Otro planteamiento diferente acerca de la recuperación del contexto para una teoría de la justicia y que es de algún modo convergente con las ideas presentadas anteriormente, se encuentran en la obra de Hunyadi, menos 
conocido todavía por el público latinoamericano, salvo por un libro disponible en castellano que recoge una parte de sus investigaciones actuales, La tiranía de los modos de vida. Doctorado en Filosofía en Ginebra con una tesis dirigida por J.M. Ferry, ha estudiado en detalle el pensamiento moral habermasiano, el cual considera que se encuentra en la base de su pensamiento y que le ha influido, pero del cual ha ido tomando paulatinamente distancia después de haber traducido al francés sus textos sobre moral y desde allí levantar sus propias premisas filosóficas.

En la óptica de Hunyadi (comunicación personal, Seminario sobre Justicia Contextual en Temuco, marzo 29, 2018), la discusión habermasiana sobre la justicia toma la forma de una discusión principal sobre los derechos humanos. Todo se relaciona en Habermas a la cuestión de la producción de legitimidad en torno a la pregunta: «¿Qué derechos fundamentales de los ciudadanos libres e iguales deberían otorgarse mutuamente si desean regular su vida en común de manera legítima con los medios del derecho positivo?». A partir de allí desarrolla su tesis de la cooriginariedad de la autonomía cívica - participación - y de la autonomía privada — libertades de acciónPero esta fundación intersubjectivista de la ley está al servicio de un marco normativo centrado en los derechos individuales, como lo demuestra el hecho de que la «Asamblea General» que se imagina Habermas — es la parte superior de la construcción institucional cosmopolita mundial- está encargado de enunciar los «parámetros de justicia» a los cuales todos los Estados deben conformarse, a saber, la consideración de los ciudadanos como individuos libres e iguales. En esta visión individualista-intersubjetivista la corrección del procedimiento de legitimación siempre asegura en Habermas la justicia del orden normativo producido.

Lo que busca establecer la teoría social de Habermas son las condiciones de imparcialidad y por lo tanto de legitimidad en las que se puede comprometer una cooperación social a pesar de las diferencias que separan a los actores en cuanto a las cuestiones éticas. Esta legitimidad puede garantizarse mediante reglas reconocidas intersubjetivamente, éstas definen las expectativas de comportamiento recíproco desplegándose a un nivel pronominal más inclusivo que el nivel en el que los actores divergen efectivamente (Hunyadi, 2015a, p. 87).

La crítica principal de Hunyadi respecto de la teoría habermasiana de la justicia apunta, específicamente, a las limitaciones de la propuesta de 
justificación de los principios y de los precarios resultados respecto del poder contrafáctico de los contextos, señala las limitaciones del planteo teórico basado en la acción comunicativa de Habermas, y apoyándose en las ideas de J.M. Ferry afirma:

Las cuestiones de justicia conciernen, entonces, de una manera general, a la regulación de los conflictos entre partes en litigio; es entonces el litigio mismo que, desde que se trata de regularlo imparcialmente, obliga a salir de su perspectiva propia y a tomar en cuenta el punto de vista antagónico. La resolución de los conflictos obliga, por tanto, a una especie de salto pronominal de la primera persona exclusiva a la primera persona inclusiva. No es en efecto más que a partir de este punto de vista abierto que podrá encontrarse una solución imparcial. La justicia no es, en efecto, la solución misma (aunque ella se dirá justa, obviamente), sino el hecho mismo de acceder por esta vía pronominal, es decir, constituyendo un Nosotros inclusivo a partir de una pluralidad siempre potencialmente conflictiva (Hunyadi, 2015b, p. 86).

En otras palabras, el principal cuestionamiento que hace el filósofo suizo tiene que ver con la respuesta política que hace Habermas, dejando de [176 ] lado los grandes temas de la filosofía social: «Es por eso que su filosofía social, sea lo que sea, realmente se disuelve en la filosofía política, abandonando su ambición inicial, que era establecer los criterios para una teoría crítica de la sociedad» (Hunyadi, 2015a, p. 93).

Volviendo a lo que interesa específicamente en este trabajo, Hunyadi no resulta convencido de las referencias contenidas en la teoría habermasiana de la justicia a la experiencia social de los actores y considera que su planteamiento es propio de un contextualismo débil. Al contrario, quiere avanzar en la elaboración de un contextualismo fuerte que se apoya en la idea de «la fuerza normativa que pueden alcanzar los contextos» (Hunyadi, 2015a, p. 322). Esta noción amplia de contexto alude al conjunto de lo que está disponible para el actor, para ayudarle a desplegar un contenido normativo: el contexto, por consiguiente, según Hunyadi, «es un stock de contenidos disponibles, permitiendo oponer contrafácticamente a lo que es un caso y de lo que se encuentra actualmente desactivado de lo que se podría o querría según fuera la situación» (p. 340). Es decir, un contexto alude a todo el conjunto normativo en el que los seres humanos nos reconocemos y es el transfondo moral y político a partir del cual desplegamos todas nuestras acciones individuales y colectivas. En este sentido, la teoría del discurso, siendo 
plenamente intersubjetivista, no logra salir de una tradición individualista, y esto lo conduce a repensar de otro modo la teoría moral y política contextual, o como prefiere decirle Hunyadi en una cierta complicidad con el planteo de Forst, es decir, partir de un pensamiento acerca del individualismo no individualista.

\section{Conclusión}

En los tres autores principalmente referidos existen convergencias y divergencias que se han abordado a partir de los trabajos citados, a la espera de un trabajo investigativo más minucioso que está en proceso. La primera cuestión que resulta patente es que estas concepciones discursivas de la justicia tienen un marcado carácter intersubjetivista y se derivan del mismo enfoque comunicativo en los que se basa la teoría de la justicia de Habermas, y que son comentadas, cuestionadas y enriquecidas por Forst y Hunyadi; pero, a su vez, los supuestos comunes de tales teorías determinan propuestas diferentes en el campo de la filosofía política y social y de las formas de comprender la moral fundamental en sus relaciones con las ciencias sociales críticas.

La problematización del contexto en su sentido fuerte y débil, que aparece en Forst y en Hunyadi, está ligado a una apropiación crítica de las tesis habermasianas; empero, a medida que se escarba en los presupuestos relativos a la justicia van apareciendo notables diferencias y varios matices categoriales. Lo que suscita interrogantes en Forst y Hunyadi acerca del modo crítico de reconstruir una teoría de la justicia apropiada para entender las sociedades contemporáneas es lo que lleva a ambos a mediar entre Habermas y Honneth. Frente al primero, Forst se desliga de un eventual énfasis idealista de la justificación y, frente al segundo, se opone a la centralidad de la idea del reconocimiento social de una identidad valiosa. Por ello se propone más bien una vía media, como lo propone explícitamente, es decir, la justificación de las normas puede convertirse en una práctica discursiva y la pregunta de por qué ella está ausente en muchos ámbitos sociales.

En este sentido, el enfoque de Forst, aunque prosigue muchas de las consignas de la ética del discurso, lo conduce a discrepar con Honneth $-\mathrm{y}$ con Fraser-, de modo que su propuesta acerca de la justicia, inspirada fuertemente en el principio de justificación habermasiano, busca superar los impases, conectando con un pluralismo diagnóstico y evaluativo que evite los escollos de la fundamentación normativa y que permita renovar la perspectiva de la teoría crítica que cuestiona las asimetrías del poder en las sociedades de 
nuestro tipo — y más allá de ella—. La postura de Forst se concentra así en una teoría crítica de la política y no en una teoría de la justicia como análisis de la sociedad, lo cual resulta de gran utilidad para pensar las asimetrías sociales de cara a un principio de justificación.

Hunyadi aceptó en principio el punto de partida de Habermas y Forst, ya que ambos enfoques tienen dos características similares: a) la voluntad de relacionarse con la experiencia social de los actores y b) su focalización no individualista de los derechos individuales. Empero, considera que el modo de interpretar el liberalismo y la teoría crítica termina unido a una visión apologética de los derechos individuales. Es por eso que Hunyadi insiste con ahínco que hoy necesitamos una institución correctiva —no fundacional- . La teoría discursiva de la ética — «pequeña ética» en Hunyadi- no es estructuralmente capaz de responder a los desafíos sistémicos planteados hoy por la globalización de los modos de vida, en particular del modo de vida tecnológico que impacta profundamente nuestras relaciones con la sociedad. Ni las respuestas individuales —en términos de resistencia a los modos de vida impuestos - ni las respuestas individualistas —en términos de reinterpretación del contrato social- pueden proporcionar una respuesta normativamente satisfactoria a estos efectos sistémicos. Por ello, la moralidad de la autonomía defendida por los filósofos de Frankfort favorecería mucho más la reproducción de los modos de vida que nadie ha elegido. Es por eso que necesitamos no refundar los derechos individuales, sino fundar una nueva praxis colectiva.

Estas dificultades detectadas por Hunyadi no se reducen únicamente a la re-interpretación del ideal de la justificación proveniente del legado kantiano o el recurso hegeliano a las formas de vida, sino a diferencias ostensibles y a focos específicos en el modo de entender la teoría crítica en el campo de la política, es decir, en la forma de repensar el proyecto de las Ciencias Sociales y Políticas actuales para pensar la vigencia problemática de los derechos humanos.

En ambas formas de dialogar con y contra Habermas aparecen otro tipo de ideas que no han nacido única y directamente del influjo del espíritu de Frankfurt y propician pensar la justicia no solo desde la dinámica de los Estados modernos nacientes, de los focos formalizadores ligados al pensamiento constitucional y jurídico relativo al Estado de derecho, ni solo desde la expansión del sistema económico actual, sino que se visualizan otras 
formas de abordar los principios de justicia y su vínculo con otras experiencias de injusticia y de las dinámicas de lucha por el reconocimiento, como lo esbozan desde la teoría del reconocimiento Honneth y Renault.

Por ello, se concluye que la cuestión del contexto es definitivamente central porque permite cuestionar y profundizar unos puntos centrales de la teoría habermasiana de la justicia, y permiten articular otros matices categoriales presentes en los proyectos de Honneth, Hunyadi y Forst, lo que demuestra la vitalidad de las teorías discursivas de la justicia. Para Hunyadi la teoría forstiana de la justicia toma la forma de una radicalización del principio de validación discursiva de Habermas que defiende un «derecho fundamental a la justificación». A pesar de los matices que aporta —ellos siguen siendo esencialmente de naturaleza metaética一, aquí también, piensa Hunyadi, el marco sigue siendo estrictamente individualista. Con todo, hay un acuerdo central, ambos coincidirían en un punto de partida para discusiones subsiguientes, lo esencial es la visión de un individualismo no individualista, el cual es el verdadero punto de partida de la crítica de Hunyadi.

Pensar la justicia en clave contextualizada, por último, recupera el interés por las experiencias de injusticia relativa a los diferentes conflictos, violencias e injusticias que vivimos en nuestras y en otras sociedades históricas, pero - por sobre todo - contribuye a visualizar la riqueza moral y política de los actores en sus contextos asimétricos. En este sentido, la pertinencia de una teoría contextual de la justicia es que ella ayuda a repensar de otro modo la discusión colectiva acerca de lo injusto que es siempre de carácter históricocultural, profundizando la relación controvertida entre procedimentalismo y lo que se ha entendido en oposición, a una forma substantiva.

En suma, al posibilitar reconstruir un ideal normativo de justicia que responda decisivamente a los conflictos que caracterizan el ethos histórico de nuestros países, que no han vivido necesariamente los mismos procesos históricos de la sociedad europea, se abre la renovación de la comprensión teórico-práctica del principio de justificación y del carácter contrafáctico del contexto. Esa es la principal contribución de Forst y Hunyadi de ayudar a repensar un Nosotros que asuma las asimetrías. Empero, la redefinición del contexto en su carácter político ya no es un asunto determinado solo por las ideas de filósofos europeos o norteamericanos, sino que es una perspectiva práctica vital que se engarza con la complejidad de la vida política. Tal exigencia contextual fuerte obliga a repensar los determinantes históricos 
principales que definen las asimetrías del poder en la sociedad y también de sus injusticias. Por eso el contexto sigue siendo lo que queda aún en deuda para nuestros pensadores políticos de la justicia.

\section{Referencias bibliográficas}

1. Forst, Rainer. (1994). Kontexte der Gerechtigkeit. Politische Philosophie jenseits von Liberalismus und Kommunitarismus. Frankfurt am Main: Suhrkamp.

2. Forst, Rainer. (2011). Kritik der Rechtfertigungverhaltnisse. Perspectiven einer kritischenTheorie der Politik. Berlin: Suhrkamp.

3. Forst, Rainer. (2014). Justificación y crítica. Perspectivas de una teoría crítica de la política. Madrid: Katz.

4. Habermas, Jürgen. (1992). Faktizität und Geltung. Beiträge zur Diskurstheorie des Rechts und des demokratischen Rechtsstaats. Frankfurt: Suhrkamp.

5. Habermas, Jürgen. (1996a). Die Einbeziehung des Anderen. Frankfurt am Main: Suhrkamp.

6. Habermas, Jürgen. (1996b). Politischer Liberalismus-Eine Auseinandersetzung mit Rawls. In: Die Einbeziehung des Anderen. Frankfurt: Suhrkamp.

7. Habermas, Jürgen. (2000). Aclaraciones a la ética del discurso. Madrid: Trotta.

8. Hunyadi, Mark. (2008). Morale Contextuelle, Quebec: Presses Universitaires de Laval.

[180 ] 9. Hunyadi, Mark. (2009). L'idée d'une contrefactualité contextuelle, ou: comment ne pas devoir transcender tous les contextes possibles, comme le veut Habermas? Revue Philosophique de Louvain, 107-2, pp. 319-349. https://doi.org/10.2143/ RPL.107.2.2040723

10. Hunyadi, Mark. (2012). L'homme en context. Paris: Éditions du Cerf.

11. Hunyadi, Mark. (2015a). La tiranía de los modos de vida. Sobre la paradoja moral de nuestro tiempo. Madrid: Cátedra.

12. Hunyadi, Mark. (2015b). Habermas: les limites politiques de la philosophie sociale. Esprit, 8 (8), pp. 80-93. https://doi.org/10.3917/espri.1508.0080

13. Pereira, Gustavo (ed.). (2013a). Perspectivas críticas de justicia social. Montevideo: Universidad de la República.

14. Pereira, Gustavo. (2013b). Elements of a Critical Theory of Justice. London: Palgrave MacMillan. https://doi.org/10.1057/9781137263384

15. Rawls, John. (1971). A Theory of Justice. Cambridge: Harvard University.

16. Rawls, John y Habermas, Jürgen. (1998). Debate sobre liberalismo político. Barcelona: Paidós.

17. Renault, Emmanuel. (2004). L'expérience de l'injustice. Paris: La Découverte. 
Contexto, justicia y universalidad en la filosofía política actual. Algunas críticas...

18. Salas, Ricardo. (2015). Notas para una contextualización de la justicia, desde y más allá de Frankfurt. Cuyo. Anuario de Filosofía Argentina y Americana, 32 (2), pp. 59-97.

19. Salas, Ricardo. (2016). Teorías contemporáneas del reconocimiento. Revista Atenea, 514, pp. 81-95.

20. Sauerwald, Gregor y Salas, Ricardo (eds.). (2017). La cuestión del reconocimiento en América Latina. Problemas y perspectivas de la teoría del reconocimiento de A. Honneth. Berlin: Lit.

21. Velasco, Juan Carlos. (1999). Introducción. Orientar la acción. La significación política de la obra de Habermas. En: Habermas, Jürgen. La inclusión del otro. Estudios de teoría política (pp. 11-22). Barcelona y Buenos Aires: Paidós. 